\title{
Cinémas
}

Revue d'études cinématographiques

Journal of Film Studies

\section{Réal La Rochelle, Opérascope. Le film-opéra en Amérique, Montréal, Triptyque, 2003, 428 p.}

\section{Frédéric Dallaire}

Volume 15, numéro 2-3, printemps 2005

Cinélekta 5

URI : https://id.erudit.org/iderudit/012326ar

Aller au sommaire du numéro

Éditeur(s)

Cinémas

ISSN

1181-6945 (imprimé)

1705-6500 (numérique)

Découvrir la revue

Citer ce compte rendu

Dallaire, F. (2005). Compte rendu de [Réal La Rochelle, Opérascope. Le film-opéra en Amérique, Montréal, Triptyque, 2003, 428 p.] Cinémas, 15(2-3),

189-197. d'utilisation que vous pouvez consulter en ligne.

https://apropos.erudit.org/fr/usagers/politique-dutilisation/ 
Réal La Rochelle, Opérascope. Le film-opéra en Amérique, Montréal, Triptyque, 2003, 428 p.

Voici une somme, un livre faisant la synthèse de plusieurs années de réflexion sur les différents aspects sonores du cinématographe. Les rapports entre l'opéra et le cinéma, ainsi qu'un genre né de cette rencontre, la comédie musicale américaine, sont déjà l'objet d'études approfondies ${ }^{1}$. L'ouvrage de Réal La Rochelle tente d'inscrire le musical dans une perspective qui transcende la notion de genre. Pour ce faire, l'auteur base sa méthode sur un entrelacement de considérations historiques et techniques, d'analyses filmiques, de commentaires critiques et d'interviews avec les cinéastes François Girard et Alain Resnais. Tous ces éléments s'intègrent ultimement dans une réflexion sur la musicalité filmique. La Rochelle tente d'évaluer le rôle de l'opéra dans l'élaboration d'une conception créative de la bande sonore. L'énorme quantité d'informations historiques et critiques communiquées par l'auteur donne au lecteur une impression de boulimie intellectuelle. Ne voulant rien oublier, il a tenté de tout inclure dans son ouvrage. Au-delà de cette tentative de "musicaliser » le texte grâce à un amalgame hétérogène de considérations fondamentales et (souvent) secondaires, il nous semble possible de retracer les bases intellectuelles de ce long voyage de 428 pages.

Lors de l'apparition du cinématographe, l'opéra traditionnel européen s'impose déjà depuis plus de trois siècles comme une manifestation spectaculaire de "l'art total». À cette époque, Thomas Edison tente de synchroniser un appareil de reproduction du mouvement avec un phonographe (p. 110). «Le film est [donc] imaginé et reçu, dès sa création industrielle, comme sonore, musical. Tel un opéra" (p. 11-12). Les possibilités offertes par l'enregistrement mécanique de l'image et du 
son ont suscité maint discours sur la démocratisation des grandes ouvres opératiques européennes. Tout un chacun peut désormais s'offrir une projection cinématographique mettant en vedette les grands chanteurs et orchestres de l'époque. Cette possibilité donna naissance à un courant d'opéra filmé. Si, en Europe, on utilise le médium audiovisuel pour garder trace des grandes performances opératiques, en Amérique du Nord, on semble rejeter cette pratique. Mises à part quelques exceptions, "l'opéra filmé n'existe pas [en Amérique]» (p. 13). Or, c'est de ce côté de l'Atlantique que l'opéra aura une influence dépassant largement la reproduction des formes traditionnelles européennes. Afin de définir "ce nouveau type de film lyrique» (p. 15), l'auteur utilise l'expression "opérascope». Celle-ci "peut marquer emblématiquement le lieu, l'objet et la stylistique d'ensemble de l'opéra dans les cinémas nord-américains " (p. 13). Il semble que les créateurs états-uniens et canadiens se soient approprié les codes opératiques afin de les transformer en les intégrant au langage cinématographique, donnant ainsi naissance à une forme nouvelle d'art audiovisuel. Le livre de La Rochelle propose donc, "au moyen d'une série de promenades, [...] de décrire les contours singuliers du film-opéra [et] d'analyser quelques films et créateurs qui en ont matérialisé les rêves et les visées" (p. 14).

Le projet est vaste et ambitieux puisque, si La Rochelle s'attarde surtout à la comédie musicale américaine, il s'intéresse aussi aux productions du Canada anglais et du Québec. Comme le souligne François Thomas ${ }^{2}$, la principale caractéristique unissant ces cinématographies fort différentes est qu'elles sont "alimentées par la même nécessité d'inventer une relation autre à la musique" (p. 399). Ce désir d'innovation traduit une conception du cinéma comme art visuel et sonore. Autant Kurt Weil, Maurice Blackburn que Michel Fano exprimeront cette idée à l'aide de formules complémentaires : "film opéra du futur ", "opéra audiovisuel ", " nouvel opéra audiovisuel " (p. 15). "Ces diverses appellations [...] sont nées de la conviction que le film-opéra moderne, véritablement novateur, surgirait du potentiel expressif des sons et images filmiques, et s'éloignerait graduellement, parfois abruptement, des formes 
dramatiques musicales de l'opéra occidental » (p. 15). Si l'Amérique du Nord constitue le terrain d'étude de La Rochelle, c'est justement parce que le poids de la tradition européenne n'était pas assez lourd pour imposer ses conventions dans une contrée à la recherche d'une culture intégrante, reflétant le modernisme nord-américain. C'est en Amérique que pourra naître un rapport à la musique véritablement nouveau et typiquement cinématographique.

Cette façon de penser la musique va remettre en question la hiérarchisation classique parole-musique-bruit, et permettre l'élaboration de ce que Michel Fano appelle "un continuum sonore à épaisseur sémantique variable» (p. 22). Autant les bruits que les paroles seront considérés comme des objets sonores devant s'intégrer dans une construction sonore globale, où l'on structure «toute la bande sonore comme une œuvre musicale électroacoustique» (p. 253). La Rochelle découvre les premiers balbutiements de cette idée dans les musicals américains. Il en cherchera toutefois l'aboutissement dans le cinéma indépendant des majors et, en cela, il prend ses distances du courant théorique dominant, qui privilégie l'étude des conventions et innovations de la comédie musicale. Cette stratégie - ayant comme base la notion de genre - ne rend pas compte de la diversité et de l'ampleur de la révolution opérée par le film-opéra sur notre conception du cinéma.

\footnotetext{
Il importe de reconnaître que le musical est plus large et plus englobant, que les conventions du genre flottent en quelque sorte dans un cosmos plus vaste qui permet leur éclatement, leur dépassement quand le genre s'éteint. [...] Cet espace hors genre est celui du filmopéra, ouvert à toutes les modulations (p. 29).
}

Le livre parcourt donc un vaste champ d'études, celui de l'opérascope, qui se tient aux limites d'un genre cinématographique, le musical, et qui tend vers une conception novatrice de la musicalité filmique, le continuum sonore.

Afin de bien comprendre le film-opéra, il est nécessaire de remédier à une certaine pauvreté perceptive et théorique du phénomène sonore: «Un des grands paradoxes de l'audiovisuel 
contemporain est qu'il ne s'entend pas, ou du moins qu'il s'entend mal»(p. 35). En effet, l'homme contemporain a développé un culte pour l'image, une fascination pour les éléments visuels qui se traduit par une pensée "abusivement iconique" (p. 37). "Si bien que le fait sonore du filmique, depuis ses origines dans le Kineto-phonograph d'Edison, a été laissé pour compte ou secondarisé dans l'histoire, l'esthétique, le langage et l'archivistique du cinéma» (p. 37). Selon l'auteur, il faut renverser ce mouvement, ne serait-ce que pour se donner les outils permettant une analyse juste des phénomènes audiovisuels. Le but poursuivi est de redonner aux occurrences sonores la place qui leur revient. Il faut permettre aux individus de comprendre l'importance du son dans le monde contemporain et les rendre sensibles aux fluctuations sonores qui restent inaudibles lorsqu'elles sont submergées par nos réflexes visuels dominants. Le terme "cinématographie" étant symptomatique de cette prédominance de l'iconique sur l'audible, La Rochelle propose le néologisme "cinéphonographie» (p. 38). Ce vaste champ d'études pratiquement inexploré pourrait trouver ses assises théoriques dans le corpus radiophonique: "Ce qu'une démarche cinéphonographique suggère, quand on veut éclaircir la donnée sonore filmique en profondeur, ce sont des prolégomènes en radio-phonographie. D'abord mieux écouter, pour ensuite bien écouter/voir»(p. 39). L'analyse des œuvres radiophoniques du tandem Welles-Hermann (p. 195-207) et du pianiste Glenn Gould (p. 369-382) nous révèle en effet une grande créativité dans l'assemblage et l'utilisation des matériaux sonores. Dans le cas de Welles, l'utilisation de la voix (narration over) et la distribution des éléments sonores (bruits, voix, musiques) prennent place dans une cinéphonographie ingénieuse et novatrice. Gould, lui, "emprunte la musicalité filmique même, [et] se réfere au langage filmique prégnant de musique» (p. 370). Le montage, le plan et le fondu enchaîné sont autant de termes qui décrivent la démarche créative de Gould. En définitive, ces œuvres soulignent les principes d'assemblage, le rythme et les modulations «d'un cinéma qui, privé de son compagnon visuel, n'en révélera que mieux son essentielle musicalité»(p. 370). Voici l'apport principal de la 
phonographie à la recherche de La Rochelle: l'organisation du son conduit l'auditeur vers une perception musicale des matériaux, et cela devrait être le présupposé fondateur des études cinéphonographiques. Ainsi, les discours privilégiant l'analyse et l'utilisation des opérascopes comme traces ou témoins des performances opératiques - en d'autres mots, comme fantômes de l'opéra (p. 127-134) — réduisent la musicalité filmique au simple sujet filmé. L'auteur s'intéresse plutôt aux œuvres qui font de la musicalité un principe formel structurant tous les éléments du film. C'est ce qui explique son choix d'inclure dans son analyse le corpus canadien et québécois, qui semble au premier regard n’avoir aucun lien avec le musical américain.

La Rochelle propose également une lecture critique des jalons historiques du film-opéra. À la suite de plus de trente années de recherche sur la synchronisation image/son, apparaissent en 1929 les premières tentatives fructueuses de musical filmique. L'innovation ne provient pas de la simple sonorisation (le fameux $100 \%$ talkie), mais du "métissage entre musiques, paroles et décors sonores de bruits d'ambiance» (p. 73). Ce modèle se distingue autant du "parlant ininterrompu ", fréquent au théâtre et à la radio, que du «flot musical continu de l'opéra» (p. 73). En nous basant sur ces distinctions, nous pourrions dire qu'il provient plutôt du musical de Broadway, où s'enchevêtrent les scènes thêâtrales et les performances musicales. Le cinéma fournira par contre la possibilité d'élaborer le «prototype d'une bande sonore intégrale, fusionnant tous les sons possibles et leurs valeurs, les soumettant du même coup aux rythmiques des montages " (p. 73). Cette conception constitue le fondement d'un nouveau genre, tout en restant assez générale pour favoriser de multiples variations et élans créatifs. En 1929, des œuvres comme The Love Parade de Lubitsch, Applause de Mamoulian et Hallelujah de King Vidor montrent déjà la vitalité et la diversité du musical naissant.

Les années 1930 consolident les caractéristiques de ce genre grâce à «la chorégraphie et la danse [qui] ajoutent une troisième dimension à la matrice parlé-chanté déjà en place» (p. 85). Les grands studios hollywoodiens utiliseront systématiquement cette matrice, ce qui provoquera rapidement un essoufflement du nouveau genre. Les producteurs tenteront de masquer la vacuité 
du système en utilisant des artifices et en faisant de l'esbroufe, misant ainsi sur la surenchère d'éléments spectaculaires. C'est ce qui explique sans doute le triomphe du chorégraphe Busby Berkeley (chapitre 4). Sur le plan musical, cette première décennie (1929-1939) est marquée par le métissage des musiques classiques et populaires. Le musical filmique, tout comme la comédie musicale de Broadway, "prend sa source principale dans la musique afro-américaine» (p. 94). Le blues et le jazz donneront le rythme et les harmonies propres au film-opéra. Or, il serait simpliste d'opposer la tradition classique européenne à la modernité populaire américaine. Certes, le musical dénonce l'élitisme de la "Grande Culture», mais il exprime également une fascination pour la musique classique (les grands orchestres, l'instrumentation, les formes imposantes, les mélodies célèbres). Dans cette relation d'amour/haine, La Rochelle perçoit «la dynamique d'une déconstruction/reconstruction du fond patrimonial, sa métamorphose, qui passe certes par le rire et le cynisme, mais aussi par une sorte d'attendrissement naïf vis-à-vis des régénérations surprenantes et inattendues» (p. 109). Un film comme $A$ Night at the Opera (1935) permet, grâce aux gestes burlesques des frères Marx, de déconstruire les rouages de la grande culture en la poussant de son piédestal pour ensuite entamer avec elle un dialogue plus respectueux. Les interactions de l'orchestre symphonique et du groupe de Louis Armstrong et Billie Holiday dans New Orleans (1947) sont un exemple de ce métissage musical et culturel.

Le musical a donc une matrice parlé-chanté-dansé forte qui s'exprime à l'aide d'une bande sonore intégrale. Les rythmes et mélodies populaires conditionnent ses aspects narratifs et formels tout en intégrant la tradition musicale européenne. Cette identité forte est le signe d'une émancipation des procédés théâtraux grâce à un modèle spécifiquement cinématographique.

Dès la fin des années 30, le musical est assez puissant et organisé pour cesser d'illustrer le vieil opéra européen. Il s'agit plutôt de conserver le meilleur de ce patrimoine, de le faire respirer dans la même coulée que le jazz ou les autres musiques populaires, de l'intégrer là où on l'attend le moins (p. 125). 
Les années 1940 et 1950 sont le théâtre de la grandeur et de la décadence du genre. La tétralogie de Betty Comden et Adolph Green ${ }^{3}$ offre un exemple de créativité extrêmement révélateur. Ainsi, "Singin' in the Rain est un discours sur le musical hollywoodien lui-même, sur MGM, sur Hollywood et sa transformation radicale à l'arrivée du sonore [...], un film autoréflexif au sens fort du terme» (p. 47). Cette tendance à prendre pour sujet sa propre réalité est une caractéristique importante de la comédie musicale. La mise en abîme permet à Comden et Green de réfléchir sur les caractéristiques du genre, sur ses vedettes, sur les mythes qu'il véhicule. On se rend alors compte de son statut contradictoire. La Rochelle cite Jane Feuer (1993) afin de décrire cette réalité inusitée:

Dans ses textes, le musical utilise un nombre de techniques généralement associées à l'art moderne. [...] Comme la peinture cubiste, les musicals fragmentent l'espace, multiplient ou divisent les corps en morceaux, en doubles ou en alter ego. Comme la danse postmoderne, les musicals mettent l'accent sur la spontanéité, la chorégraphie de groupe et les techniques de naturalisme. Comme Godard, les musicals s'adressent directement au public, ou font usage de personnages complexes et contradictoires. Comme Fellini, les musicals se promènent dans divers niveaux de réalité et sur la continuité entre des images oniriques et vivantes. Pourtant, le musical hollywoodien ne ressemble en rien à ces œuvres modernes. Le musical est formellement chauve, culturellement le plus conservateur des genres filmiques (p. 135-136).

Nous retrouvons dans la tétralogie de Comden et Green une synthèse des innovations formelles du genre tout autant que ses codes, ses clichés et ses tics. À l'intérieur du système hollywoodien, ces deux scénaristes ont retourné le musical sur lui-même, cette réflexivité représentant à la fois un accomplissement majeur et un signe de déclin. D'ailleurs, d'autres grands artistes échoueront dans leur tentative d'intégration de leur génie à la matrice hollywoodienne - Kurt Weil et Leonard Bernstein, par exemple (p. 153-178). 
Le renouvellement des créations du musical ne semblant pas pouvoir se produire à l'intérieur des studios hollywoodiens, La Rochelle met en lumière la modernité du film-opéra dans le cinéma canadien et québécois. Émancipés des contraintes du genre musical, certains cinéastes et compositeurs expérimentent dès les années 1950 de nouveaux liens entre le son et l'image. Norman McLaren et Maurice Blackburn sont les chefs de file de cette première génération de compositeurs/concepteurs sonores. La conscience des possibilités et des puissances d'une structuration intégrale de la bande-son représente une caractéristique prégnante du cinéma de plusieurs artistes. Ainsi, La Rochelle s'intéresse à Michael Snow (p. 295-305), Peter Mettler (p. 305309), Pierre Hébert (p. 310-317), Fernand Bélanger (p. 359362), Gilles Groulx (p. 362-365), Jacques Leduc (p. 351-354) et Jean Chabot (p. 356-359). Fidèles aux idées véhiculées par le compositeur électroacoustique Yves Daoust lors de son passage à l'Atelier de conception et de réalisations sonores de l'ONF (p. 249-256), ces cinéastes considèrent le son comme un élément fondamental du discours filmique. La Rochelle porte une attention particulière au montage de ces films, puisqu'il représente le fondement de la musicalité filmique (p. 349-350). Le cinéma, "art du Temps», s'inspire ici de la musique afin de créer de véritables "partitions audiovisuelles opératiques" (chapitre 16). En définitive, le cinéma canadien et québécois réalise - non sans difficultés, contradictions et approximations - des œuvres basées sur une matrice issue du musical américain, où nous voyons poindre une intégration du phénomène sonore dans une conception créatrice du discours audiovisuel.

À la fin du livre de La Rochelle, il apparaît que "le véritable opéra filmique américain, le Filmoper, c'est l'alchimie de l'image en mouvement et du son par la musicalité» (p. 386). Cette définition justifie, d'après l'auteur, la parenté entre l'opéra et le vaste corpus étudié. La question fondamentale de cet ouvrage est la suivante: "[...] y a-t-il une actualité contemporaine pour l'opéra, qu'on dit effondré depuis les années vingt [...] ?" (p. 18). La réponse est affirmative. Cette actualité passe par le médium cinématographique. "Le premier siècle du cinéma aura entre autres servi à cette passionnante renaissance: succéder à 
l'opéra scénique vieux de trois siècles et l'installer, en le renouvelant de fond en comble mais sans en renier la richesse patrimoniale, dans un lieu et avec des moyens contemporains, la cinéphonographie» (p. 387). Il resterait alors à trouver un véritable nom à cette transformation de la forme opératique. Plusieurs compositeurs et cinéastes ont hésité sur la caractérisation de cette forme d'expression artistique ${ }^{4}$. La Rochelle propose de nommer "opéra de New York" l'opérascope nord-américain (p. 396). C'est en effet à Broadway que la matrice du film-opéra fut créée. Le nouveau siècle nous réserve des mutations encore considérables de ce modèle, puisqu'on le rencontre désormais fréquemment dans le médium vidéographique. Pour La Rochelle, l'opéra n'est pas en déclin, mais on le retrouve là où l'on ne l'attendait pas, loin de la scène théâtrale: sur l'écran.

Frédéric Dallaire

Université de Montréal

\section{NOTES}

1. Les plus connues étant celles d'Altman (1992), de Feuer (1993), d'Ishaghpour (1995) et de Masson (1981).

2. Professeur à l'Université de Rennes, spécialiste d'Alain Resnais et d'Orson Welles. Il signe la postface d'Opérascope.

3. La Rochelle considère ces deux scénaristes comme de véritables auteurs. Cette idée pertinente permet une analyse éclairante de leurs quatre films majeurs, soit The Barkleys of Broadway (1949), Singin' in the Rain (1952), The Band Wagon (1953) et It's Always Fair Weather (1955).

4. Voir les citations de Kurt Weil, Leonard Bernstein, John Mauceri et Stephen Sondheim (p. 387).

\section{RÉFÉRENCES BIBLIOGRAPHIQUES}

Altman 1992 : Rick Altman, La comédie musicale hollywoodienne, Paris, Armand Colin, 1992.

Feuer 1993 : Jane Feuer, The Hollywood Musical, Bloomington/Indianapolis, Indiana University Press, 1993.

Ishaghpour 1995 : Youssef Ishaghpour, Opéra et théâtre dans le cinéma d'aujourd'hui, Paris, La Différence, 1995.

Masson 1981 : Alain Masson, Comédie musicale, Paris, Stock, 1981. 